\title{
Facial Expression Recognition using PCA Algorithm
}

\author{
Shweta Patil \\ Student Electronics, \\ Amrutvahini College, \\ Sangamner, \\ Maharashtra, India
}

\author{
S.S.Katariya \\ Associate Prof \\ Amrutvahini College, \\ Sangamner, \\ Maharashtra, India,
}

\begin{abstract}
Face is the primary focus for the identity of human. But while detecting the face one difficulty is there. How to deal with the variations in the facial expressions, lightening etc. in this paper we use the principal component analysis (PCA) algorithm for the detection of facial expression. First the eigen spaces are created with the help of eigen vectors and eigen values. With the help of this space eigen faces are created and with the help of PCA algorithm the most matching eigen face is selected. The databases of 30 persons are generated each person having 10 photographs with different expressions like happy, angry, sad, neutral etc. If any expression is not recognize then it consider as a neutral expression. The classifier used are based on Euclidian distance. Train and test databases are there but that should be in similar conditions such as distance, lightening, background etc. The results shows the accuracy of this algorithm.
\end{abstract}

\section{Keywords}

Principal component analysis, Eigen vector, Eigen values.

\section{INTRODUCTION}

For the identity of the person, face is the primary focus. Facial expression detection is nothing but the detection of the face and then recognize the expression of the detected face. There are many expressions like happy, sad, angry, neutral, disgust etc. Many algorithms gives the best result to find out the facial expressions for example,

\section{PCA Algorithm}

SVD Algorithm

\section{GABOR Algorithm}

Feature extraction means extracting certain feature points on the face like

1. Eyes

2. Nose

3. Lips

4. Eye Brows.

\section{LITERATURE SURVEY}

[1] This paper provides the knowledge of eigen faces. The mathematical formulae and operators used in the principal component analysis algorithm are describe in this paper. Eigen values and mean values are the operator used by PCA algorithm to match the most relevant eigen face. Mathematical description is provide in this paper.

[2] This paper gives the idea how principal component analysis (PCA) is superior algorithm than SVD. The comparison between this algorithm are mentioned in this paper. These algorithm are tested and evaluated with real time and JAFEE database. This paper also recognized the following emotions: sad, disgust, happy, angry, surprise.

[3] This paper is based on biometric authentication. This paper gives an idea about a system which recognize the person's expression and so that the prohibited work can be done in safe condition as this system reports the condition of person to security.

[4]. This paper gives a new idea for detecting the human face which is unknown to system and then recognize the expression. The objective of this paper is to develop the robots or machines which can work by mind. The motive of recognition of facial expression system is to detect the faces or detect the locations of faces in the image or scene. So for this purpose this paper suggest the system called as (FACS) facial action coding system. This system works automatically to detect the facial expressions.

[5] In this paper different methods for feature extraction are discussed with their performance parameter. These methods are based on the (HLAC) higher order local autocorrelation and (LBP) local binary pattern.

\section{SYSTEM ANALYSIS}

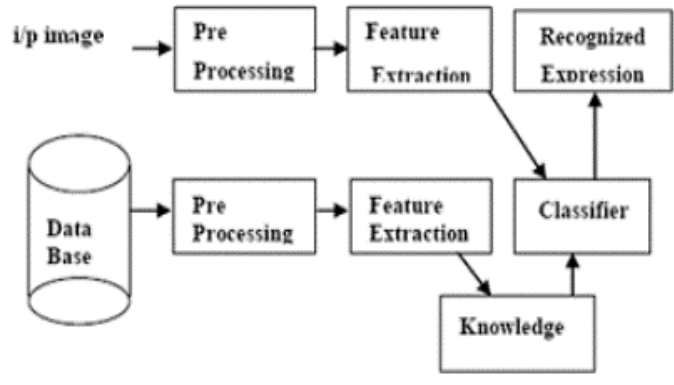

Fig: Block Diagram of Face recognition

\subsection{Explaination}

Following are the main steps in recognition process:

1) Pre-processing:

In the stage of pre -processing we try to make the input images of the testing database compatible with the developed code. For this purpose we first convert the input colored image into the gray scale values. Later we have used the low pass filter to avoid the effect of blur and to reduce the noise if any from the input image to get the exact expected results. 
2) Feature extraction:

Here we consider certain features of the face to be compared with the existing database and extract them from input face image. The features that can be extracted are as follows:

\section{Nose, Lips, Eyes, Brows}

\section{3) Database}

The database consists of the training or the reference images which is interfaced with the required software with the help of coding for the further matching with the input images. This database could be one of the standard databases or the self prepared database. The functions of pre-processing and feature extraction are also operated on the database images to obtain the average values for the sake of comparison with the values obtained by the input images

4) Knowledge:

This block consists of the required information for the classifier to compare with the training and testing images like some mean values or some thresholds or some standards to be followed while making the comparison. Also it contains a file for referring of the required expression set to be compared with some standard values.

\section{5) Classifier:}

This block uses the knowledge set which help in comparison of the vales obtained from the input images and that obtained by the database already stored and gives the recognized expression to the recognizer block to be displayed in the result.

6) Recognized expression:

This block accepts the results from the classifier and displays it into the required GUI format and also generates a text file which gives a list of the images classified into the required expression sets.

\section{PCA Algorithm:}

This approach of expression detection involves the following initialization operations:

1) First gather the set having initial face images which we called as training set.

2) Then Calculate the eigen faces from that training set images and keep the record of $M$ images which have the highest eigen values and other images can be discarded.

3) These $M$ images are called as face space. When new eigen faces are obtained then this face space can be replaced with that values.

4) Then compare the images based on eigen values which matches closly.

\begin{tabular}{|c|c|}
\hline Facial Expression & $\begin{array}{c}\text { Recognition Rate using } \\
\text { PCA }\end{array}$ \\
\hline HAPPY & 95.00 \\
\hline DISGUST & 70.00 \\
\hline SURPRISE & 85.00 \\
\hline ANGRY & 60.00 \\
\hline SAD & 90.00 \\
\hline
\end{tabular}

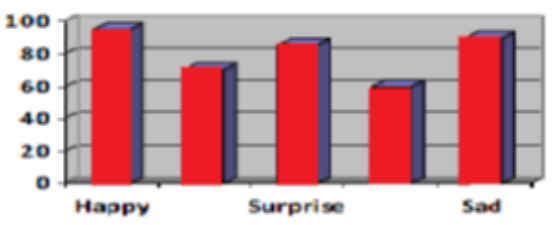

Fig: PCA efficiency for various expressions.

\section{RESULTS}

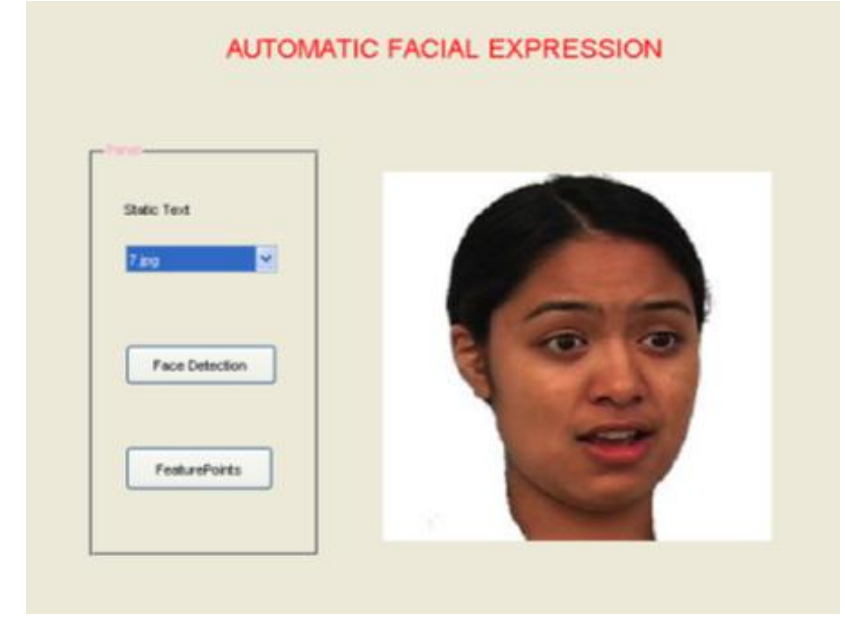

Fig: Before Face detection

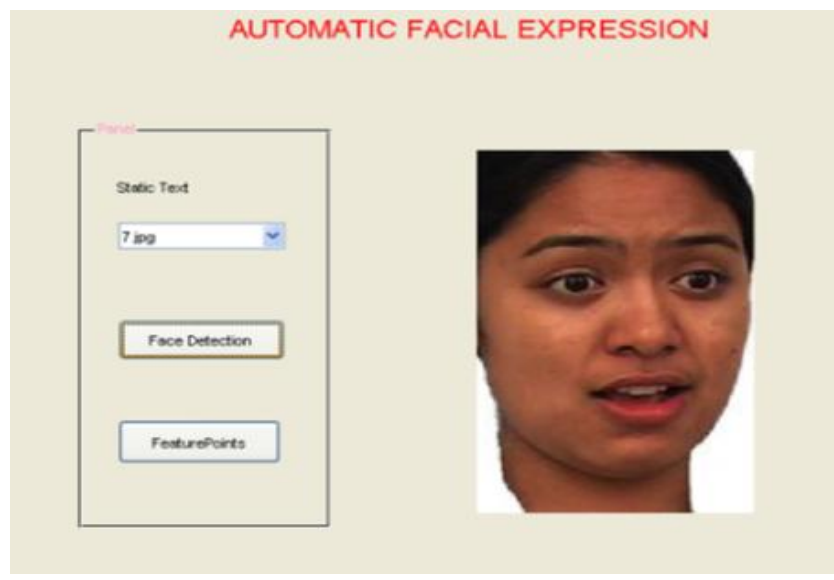

Fig: After Face detection 


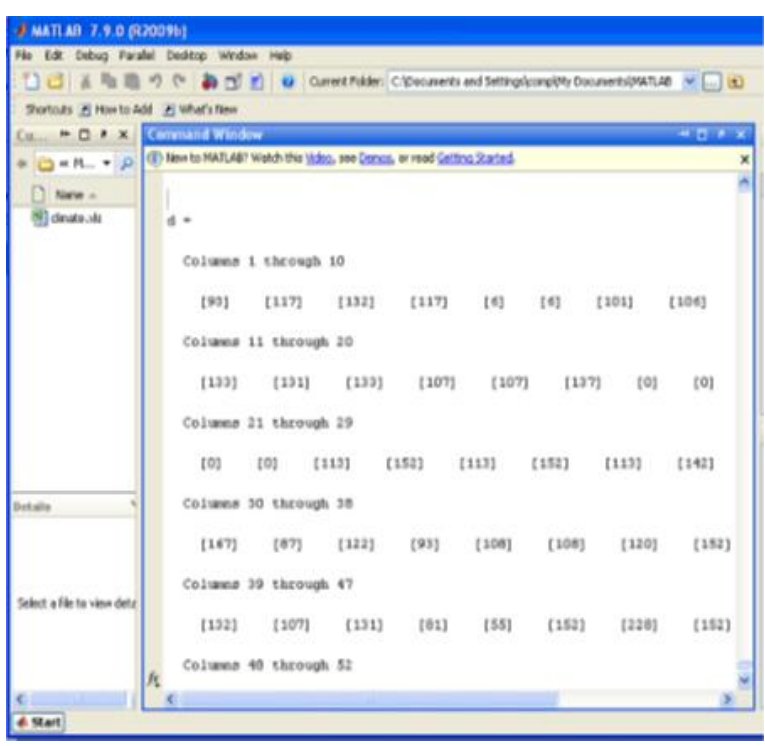

Fig: Features point calculated from the image

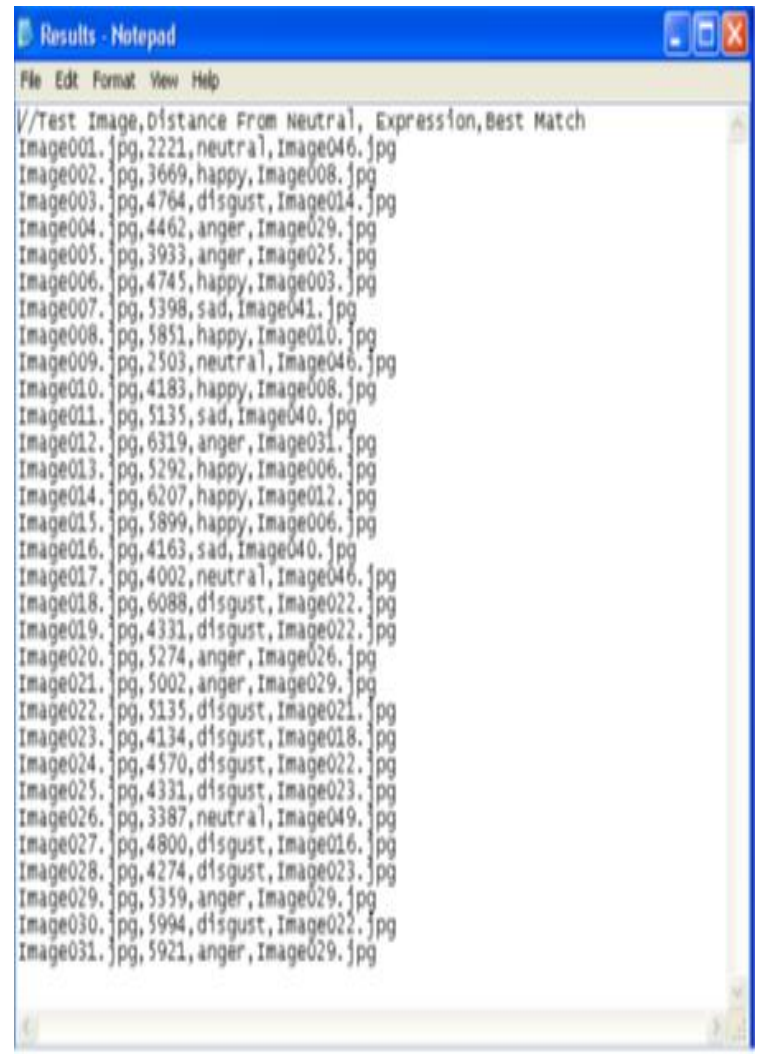

Fig: Recognized expressions of every image

\section{FUTURE SCOPE}

This application is completed on the MATLAB, in the future; the application can be performed with programming of Java or C++.In future we plan to implement this system with real time applications. Also we plan to improve the accuracy of this system.

\section{CONCLUSION}

This system is implemented for the recognition of facial expression automatically. In this first we extract the face region. In that we extracted the features like lips, nose, eyes, eye brow etc using PCA algorithm. Then we calculate the eigen vectors and compare with the real database as well as standard database. The experimental results shows the accuracy of the system on different expressions using PCA algorithm.

\section{REFERENCES}

[1] Abhishek Singh and Saurabh Kumar "Face Recognition using Principal component analysis and Eigen Face approach"

[2] "Comparative Study of Facial expression Recognition", International Journal of Computer Applications (0975 8887)Volume 13- No.1, January 2011

[3] Ashraf Abbas M. Al-modwahi, Onkemetse Sebetela, Lefoko Nehemiah Batleng, Behrang Parhizkar, Arash Habibi Lashkari "Facial Expression Recognition Intelligent security System For real Time Surveillanc"

[4]"Recognition of Facial Expression with PCA and Singular value Decomposition" International Journal of Computer Applications Volume 9U No.12, November 2010.

[5] Seyed Mehdi Lajevardi, Zahir M. Hussai " Local Feature Extraction method for Facial expression recognition", 17th European Signal Processing Conference (EUSIPCO 2009) Glasgow, Scotland, August 24-28, 2009

[6] Bartlett, M. A., Ekman, P., Hager, J. C., and Sejnowski T.,1999, "Measuring facial expressions by computer image analysis", Journal of Psychophysiology, Vol. 36, No. 2, pp.253-263.

[7] Bartlett, M. S., Donato, G., Ekman, P., Hager, J. C.,Sejnowski, T.J., 1999,"Classifying Facial Actions", IEEE Trans. Pattern Analysis and Machine Intelligence, Vol. 21,No. 10, pp. 974-989

[8] Cohn, J., Kanade, T., Lien, J., 2000, "Detection, tracking and classification of action units in facial expression",Journal of Robotics and Autonomous Systems, Vol. 31, pp.131-146.

[9] Jain, A.K., Duin R.P.W., Mao J., 2000,"Statistical Pattern Recognition: A Review", IEEE Trans. Pattern Analysis and Machine Intelligence, Vol. 22, No. 1,pp. 4-37.

[10] Pantic, M. and Rothkrantz, L., 2000, "Automatic analysis of facial expressions: The state of the art", IEEE Transactions on Pattern Analysis and Machine Intelligence, Vol. 22, No. 12, pp.1424-1445.

[11] Iyengar, P.A., Samal, A., (1992) "Automatic Recognition andAnalysis of Human Faces and Facial Expressions: A Survey", Pattern Recognition, Vol. 25, No. 1, pp. 65-77.

[12] Chellappa, R., Sirohey S., Wilson C.L., (1995) "Human andMachine Recognition of Faces: a Survey", Proc. IEEE, Vol.83, No. 5, pp. 705-741. [5] Cohen W., Fast Effective Rule Induction, In Proc. 12th international Conf. Machine Learning (ICML'95), pp 115-123, 1995. 